\title{
A Note on the Lie Algebra of the Invariants in the CBS Nonlinear Equation
}

\author{
Jose M. Cerveró \\ Fsica Teórica, Facultad de Ciencias, Universidad de Salamanca, Salamanca, Spain \\ Email: cervero@usal.es
}

How to cite this paper: Cerveró, J.M. (2018) A Note on the Lie Algebra of the Invariants in the CBS Nonlinear Equation. Journal of Modern Physics, 9, 1297-1303. https://doi.org/10.4236/jmp.2018.96078

Received: March 21, 2018

Accepted: May 22, 2018

Published: May 25, 2018

Copyright $\odot 2018$ by author and Scientific Research Publishing Inc. This work is licensed under the Creative Commons Attribution International License (CC BY 4.0).

http://creativecommons.org/licenses/by/4.0/

\begin{abstract}
In this short note, a particular realization of the vector fields that form a Lie Algebra of symmetries for the Calogero-Bogoyavleskii-Schiff equation is found. The Lie Algebra is examined and the result is a semidirect product of two Lie Groups. The structure of the semidirect product is examined through the table of commutation rules. Two reductions are made with the help of two sets of generators and the final outcome for the solution is related to the elliptic Painlevé $\mathcal{P}(\xi)$-function.
\end{abstract}

\section{Keywords}

Nonlinear Integrability, Group Theory, Weierstrass P-Elliptic Function

\section{Introduction}

The Calogero-Bogoyavleskii-Schiff Nonlinear Partial can be written as [1]:

$$
u_{x t}+u_{x} u_{x y}+\frac{1}{2} u_{x x} u_{y}+\frac{1}{4} u_{x x x y}=0
$$

The study of the integrability of this equation has been the subject of a large body of literature concerning specially the solutions obtained by means of a wealth of methods. For instance the interested reader may wish to check the references [2], [3], [4] and [5]. To achieve this goal the method of the Singular Manifold Expansion has been extensively used. Starting with the encyclopedic work of P. J. Olver in his book of Symmetries in Differential Equations [6], J. Weiss, M. Tabor and G. Carnevale [7] and John Weiss himself [8] developed the theory of the Singular Manifold Expansion and its applications. An excellent modern account of the method as well as various examples can be found in the recent review of [9]. In this note we shall review known results with the aim of finding the Lie Algebra structure using the Integrals of Motion method and 
alternative modes of finding solutions that can be applied in the future to other Nonlinear Partial Differential Equations.

\section{The Symmetries}

The vector fields of the CBS equation take the following form in $x, y, t$ and the field $u(x, y ; t)$ coordinates:

$$
\begin{gathered}
\mathbf{X}_{1}=\frac{\partial}{\partial t}+f_{1}(t) \frac{\partial}{\partial x}+\left\{2 y f_{1}^{\prime}(t)+f_{2}(t)\right\} \frac{\partial}{\partial u} \\
\mathbf{X}_{2}=f_{3}(t) \frac{\partial}{\partial x}+\frac{\partial}{\partial y}+\left\{2 y f_{3}^{\prime}(t)+f_{4}(t)\right\} \frac{\partial}{\partial u} \\
\mathbf{X}_{3}=f_{5}(t) \frac{\partial}{\partial x}+t \frac{\partial}{\partial y}+\left\{2 y f_{5}^{\prime}(t)+f_{6}(t)+x\right\} \frac{\partial}{\partial u} \\
\mathbf{X}_{4}=t \frac{\partial}{\partial t}+\left\{f_{7}(t)+\frac{x}{2}\right\} \frac{\partial}{\partial x}+\left\{2 y f_{7}^{\prime}(t)+f_{8}(t)-\frac{u}{2}\right\} \frac{\partial}{\partial u} \\
\mathbf{X}_{5}=\left\{f_{9}(t)-\frac{x}{2}\right\} \frac{\partial}{\partial x}+y \frac{\partial}{\partial y}+\left\{2 y f_{9}^{\prime}(t)+f_{10}(t)+\frac{u}{2}\right\} \frac{\partial}{\partial u} \\
\mathbf{X}_{6}=\frac{t^{2}}{2} \frac{\partial}{\partial t}+\left\{f_{11}(t)+\frac{x t}{4}\right\} \frac{\partial}{\partial x}+\frac{y t}{2} \frac{\partial}{\partial y}+\left\{2 y f_{11}^{\prime}(t)+f_{12}(t)-\frac{u t}{4}+\frac{x y}{2}\right\} \frac{\partial}{\partial u}
\end{gathered}
$$

To find the form of the arbitrary functions $f_{i}(t)$ where $i=1,2,3, \cdots, 12$, we have to minimize the number of vector fields by using the commutators of Table 1 which give rise to the following set of Ordinary Differential Equations:

$$
\begin{gathered}
f_{4}^{\prime}(t)=2 f_{1}^{\prime}(t) ; \quad f_{5}^{\prime}(t)=f_{3}(t) ; \quad 2 t f_{1}^{\prime}(t)-f_{1}(t)=f_{6}^{\prime}(t)-f_{4}(t) \\
t f_{2}^{\prime}(t)+\frac{3}{2} f_{2}(t)=t f_{8}^{\prime}(t) f_{1}^{\prime}(t)+\frac{1}{2} f_{1}=f_{7}^{\prime}(t) ; \quad f_{9}^{\prime}(t)=\frac{1}{2} f_{1}(t) \\
f_{10}^{\prime}(t)+\frac{1}{2} f_{2}(t)=0 ; \quad \frac{1}{2}\left\{t f_{2}^{\prime}(t)+\frac{1}{2} f_{2}(t)\right\}=f_{12}^{\prime}(t)-f_{8}-\frac{1}{2} f_{10}(t) \\
\frac{1}{2} t\left\{t f_{1}^{\prime}(t)-\frac{1}{2} f_{1}(t)\right\}=f_{11}^{\prime}(t)-f_{7}-\frac{1}{2} f_{9}(t) ; \quad t f_{4}^{\prime}(t)+\frac{1}{2} f_{4}=2 f_{7}^{\prime}(t)
\end{gathered}
$$

Table 1. Lie algebra $G$ formed by $\mathrm{G}_{1}, \mathrm{G}_{2}, \mathrm{G}_{3}, \mathrm{D}_{1}, \mathrm{D}_{2}$ and $\mathrm{D}_{3}$.

\begin{tabular}{ccccccc}
\hline Lie Algebra & $\mathbf{G}_{1}$ & $\mathrm{G}_{2}$ & $\mathrm{G}_{3}$ & $\mathrm{D}_{1}$ & $\mathbf{D}_{2}$ & $\mathbf{D}_{3}$ \\
\hline $\mathbf{G}_{1}$ & 0 & 0 & $\mathbf{G}_{2}$ & $\mathrm{G}_{1}$ & 0 & $\mathbf{D}_{1}+\frac{1}{2} \mathbf{D}_{2}$ \\
$\mathbf{G}_{2}$ & 0 & 0 & 0 & 0 & $\mathbf{G}_{2}$ & $\frac{1}{2} \mathbf{G}_{3}$ \\
$\mathrm{G}_{3}$ & $-\mathrm{G}_{2}$ & 0 & 0 & $-\mathrm{G}_{3}$ & $\mathrm{G}_{3}$ & 0 \\
$\mathbf{D}_{1}$ & $-\mathrm{G}_{1}$ & 0 & $\mathrm{G}_{3}$ & 0 & 0 & $\mathbf{D}_{3}$ \\
$\mathbf{D}_{2}$ & 0 & $-\mathrm{G}_{2}$ & $-\mathrm{G}_{3}$ & 0 & 0 & 0 \\
$\mathbf{D}_{3}$ & $-\left(\mathbf{D}_{1}+\frac{1}{2} \mathbf{D}_{2}\right)$ & $-\frac{1}{2} \mathbf{G}_{3}$ & 0 & $-\mathbf{D}_{3}$ & 0 & 0 \\
\hline
\end{tabular}




$$
\begin{gathered}
\left\{t f_{4}^{\prime}(t)+\frac{1}{2} f_{4}(t)\right\}=2 f_{11}^{\prime}(t)-\frac{1}{2} f_{6}(t) ; \quad 2 f_{9}^{\prime}(t)-\frac{1}{2} f_{4}(t)=0 \\
\left\{t f_{6}^{\prime}(t)-\frac{1}{2} f_{6}(t)\right\}=2 t f_{7}^{\prime}(t)-f_{7}(t) ; \quad\left\{t f_{6}^{\prime}(t)+\frac{1}{2} f_{6}(t)\right\}=4 f_{11}^{\prime \prime}(t) \\
2 t f_{8}^{\prime}(t)-f_{8}(t)=\frac{1}{2} f_{6}(t) ; \quad 2 t f_{9}^{\prime}(t)-f_{9}(t)=f_{7}(t) ; \quad 2 t f_{10}^{\prime}(t)+f_{10}(t)+f_{8}(t)=0 \\
t f_{11}^{\prime}(t)-32 f_{11}(t)=\frac{t}{4}\left\{2 t f_{7}^{\prime}(t)-f_{7}(t)\right\} ; \quad 2 t f_{12}^{\prime}(t)-f_{12}(t)=t\left\{t f_{8}^{\prime}(t)+\frac{1}{2} f_{8}(t)\right\} \\
\frac{t}{2}\left\{2 t f_{9}^{\prime}(t)-f_{9}(t)\right\}=f_{11}(t) ; \quad \frac{t}{2}\left\{2 t f_{10}^{\prime}(t)+f_{10}(t)\right\}=-f_{12}(t)
\end{gathered}
$$

The following functions form a non-unique solution of the above system of Ordinary Differential Equations:

$$
\begin{aligned}
& f_{1}(t)=\frac{1}{2} f_{4}(t)=f_{10}(t)=t^{-\frac{1}{2}} ; \quad f_{2}(t)=t^{-\frac{3}{2}} ; \quad f_{9}(t)=t^{\frac{1}{2}} \\
& f_{3}(t)=f_{5}(t)=f_{6}(t)=f_{7}(t)=f_{8}(t)=f_{11}(t)=f_{12}(t)=0
\end{aligned}
$$

With these solutions the vector fields are easily shown to be:

$$
\begin{gathered}
\mathbf{G}_{1}=\frac{\partial}{\partial t}+t^{-\frac{1}{2}} \frac{\partial}{\partial x}+t^{-\frac{3}{2}}(1-y) \frac{\partial}{\partial u} \\
\mathbf{G}_{2}=\frac{\partial}{\partial y}+2 t^{-\frac{1}{2}} \frac{\partial}{\partial u} \\
\mathbf{G}_{3}=t \frac{\partial}{\partial y}+x \frac{\partial}{\partial u} \\
\mathbf{D}_{2}=\left\{t \frac{\partial}{\partial t}+\frac{x}{2} \frac{\partial}{\partial x}-\frac{u}{2} \frac{\partial}{\partial u}\right. \\
\left.t^{\frac{1}{2}}-\frac{x}{2}\right\} \frac{\partial}{\partial x}+y \frac{\partial}{\partial y}+\left\{t^{-\frac{1}{2}}(1+y)+\frac{u}{2}\right\} \frac{\partial}{\partial u} \\
\mathbf{D}_{3}=\frac{t^{2}}{2} \frac{\partial}{\partial t}+\frac{x t}{4} \frac{\partial}{\partial x}+\frac{y t}{2} \frac{\partial}{\partial y}+\frac{1}{4}\{2 x y-u t\} \frac{\partial}{\partial u}
\end{gathered}
$$

\section{The Lie Algebra Structure}

As can easily be seen the Invariants [10] can be divided into two different set of elements called $\mathcal{G}:\left\{\mathbf{G}_{1}, \mathbf{G}_{2}, \mathbf{G}_{3}\right\}$ and $\mathcal{D}:\left\{\mathbf{D}_{1}, \mathbf{D}_{2}, \mathbf{D}_{3}\right\}$ each of them possesing a Lie Algebra structure with commutation relations being of the form:

$$
\begin{array}{rll}
{\left[\mathbf{G}_{1}, \mathbf{G}_{2}\right]=0 ;} & {\left[\mathbf{G}_{2}, \mathbf{G}_{3}\right]=0 ;} & {\left[\mathbf{G}_{3}, \mathbf{G}_{1}\right]=-\mathbf{G}_{2}} \\
{\left[\mathbf{D}_{1}, \mathbf{D}_{2}\right]=0 ;} & {\left[\mathbf{D}_{2}, \mathbf{D}_{3}\right]=0 ;} & {\left[\mathbf{D}_{3}, \mathbf{D}_{1}\right]=-\mathbf{D}_{3}}
\end{array}
$$

Although the form of the Lie Algebras seems at the first sight to be the same, this conclusion is wrong as the first one is isomorphic to a sort of Non-Extended Galilei Lie Algebra $\mathcal{G}$ [11] and the second one is isomorphic to a Lie Algebra of Dilatations in one of the spatial coordinates $\mathcal{D}$ [12]. Both Lie Algebras form 
the entire Lie Algebra of Symmetries $G$ as a Semi-Direct Product which it is now written as:

$$
G=\mathcal{G} \oslash \mathcal{D}
$$

Te rest of the entire Goup of Symetries has the following Lie Algebra that contains the rest of the information on the Semi-Direct Product:

$$
\begin{gathered}
{\left[\mathbf{G}_{1}, \mathbf{D}_{1}\right]=\mathbf{G}_{1} ; \quad\left[\mathbf{G}_{1}, \mathbf{D}_{2}\right]=0 ; \quad\left[\mathbf{G}_{1}, \mathbf{D}_{3}\right]=\mathbf{D}_{1}+\frac{1}{2} \mathbf{D}_{2}} \\
{\left[\mathbf{G}_{2}, \mathbf{D}_{1}\right]=0 ; \quad\left[\mathbf{G}_{2}, \mathbf{D}_{2}\right]=\mathbf{G}_{2} ; \quad\left[\mathbf{G}_{2}, \mathbf{D}_{3}\right]=\frac{1}{2} \mathbf{G}_{3}} \\
{\left[\mathbf{G}_{3}, \mathbf{D}_{1}\right]=-\mathbf{G}_{3} ; \quad\left[\mathbf{G}_{3}, \mathbf{D}_{2}\right]=\mathbf{G}_{3} ; \quad\left[\mathbf{G}_{3}, \mathbf{D}_{3}\right]=0}
\end{gathered}
$$

\section{The Solutions}

As is well known each symmetry and its correspondent differential invariant are related to a solution of the CBS Equation. Also any linear combination of the Invariants corresponds to a given change of variables (often called "reduction") which lowers the number of variables from three to one and/or from three to two, depending of the form of the generator.

1) An interesting and trivial combination of Invariants is to consider only $\mathbf{G}_{1}$ as the invariant that leads just to the reduction that we shall analyze below [3]. We then consider a reduction with the invariant:

$$
\mathbf{G}_{1}=\frac{\partial}{\partial t}+t^{-\frac{1}{2}} \frac{\partial}{\partial x}+t^{-\frac{3}{2}}(1-y) \frac{\partial}{\partial u}
$$

that gives as a result the change of independent and dependent variables in the form: $\eta=x-2 y^{\frac{1}{2}}-2 t^{\frac{1}{2}}$ and $W(z, y)=u(x, y, t)+2 t^{-\frac{1}{2}}(1-y)$ and the CBS equations is then reduced to the Ordinary Nonlinear Differential Equation written below with one variable which has been found through the method of characteristics [6]. The details can be found in [3] and the result is:

$$
W^{\prime \prime \prime \prime}+6 W^{\prime} W^{\prime \prime}=0
$$

where the primes indicate the derivatives with respect to the $\eta$ independent variable.

To solve this equation we first consider the following ODE:

$$
\frac{1}{2} \psi^{\prime 2}+\psi^{3}=c_{1} \psi+c_{2}
$$

Deriving twice this new equation we easily find:

$$
\psi^{\prime \prime \prime}+6 \psi \psi^{\prime}=0
$$

The initial function $W(\eta)$ is related to $\psi(\eta)$ simply by $\psi=W^{\prime}$ where the prime denotes derivative with respect to the independent variable $\eta$ as can be trivially checked. Now let us look for a solution of the equation for $\psi$. In order to solve the equation for $\psi$ we should consider the following identity involving the Weierstrass $\mathcal{P}$-Function: 


$$
\frac{1}{2} \mathcal{P}^{\prime 2}=4 \mathcal{P}^{3}-g_{2} \mathcal{P}-g_{3}
$$

that coincides with:

$$
\frac{1}{2} \psi^{\prime 2}+\psi^{3}=c_{1} \psi+c_{2}
$$

if and only if:

$$
\psi(\eta)=\mathcal{P}\left(\frac{i}{2} \eta\right)
$$

and $g_{2}=4 c_{1}$ and $g_{3}=4 c_{2}$. The symbol $\eta$ stands for the independent variable with respect to which we have performed all the derivatives. The last step is to find $W(\eta)$. Due to the fact that $W^{\prime}=\psi$ we obtain trivially:

$$
W(\eta)=\int_{-\infty}^{\eta} \mathcal{P}\left(\frac{i}{2} \eta\right) \mathrm{d} \eta=\frac{i}{2} \int_{-\infty}^{\eta} \mathcal{P}(\xi) \mathrm{d} \xi
$$

where $\xi=\frac{i}{2} \eta$. The integral of the Weierstrass $\mathcal{P}$-Function cannot be expressed as a closed function but rather as a series of powers in the independent variable $\xi$. The interest reader can learn about this integral in the book: In particular one can find there the expression of the integral of $\mathcal{P}(\xi)$ (see [13] and formula $1036.50 \mathrm{on}$ it) as:

$$
\int_{-\infty}^{\eta} \mathcal{P}(\xi) \mathrm{d} \xi=-\frac{1}{\xi}+\frac{g_{2}}{60} \xi^{3}+\frac{g_{3}}{140} \xi^{5}+\frac{g_{2}^{2}}{8400} \xi^{7}+\frac{g_{2} g_{3}}{18480} \xi^{9}+\left.\cdots\right|_{-\infty} ^{\eta}
$$

The result for our case using the expression for the argument $\xi=\frac{i}{2} \eta$ and the fact that $g_{2}=4 c_{1}$ and $g_{3}=4 c_{2}$ is:

$$
\begin{aligned}
W(\eta)= & \frac{4}{\eta}-\frac{c_{1}}{60} \eta^{3}+\frac{c_{2}}{560} \eta^{5}-\frac{c_{1}^{2}}{33600} \eta^{7}+\frac{c_{1} c_{2}}{295680} \eta^{9}-\frac{196 c_{1}^{3}+375 c_{2}^{2}}{2627625} \eta^{11} \\
& +\frac{c_{1}^{2} c_{2}}{38438400} \eta^{13}-\left\{\frac{2 c_{1}^{4}}{1243125}+\frac{8 c_{1} c_{2}^{2}}{595595}\right\}\left(\frac{\eta}{2}\right)^{15}+\cdots
\end{aligned}
$$

The solution becomes a pure real function for all $\eta \in \mathcal{R}$. One interesting property of the solution given as an expansion of the Weierstrass $\mathcal{P}(\xi)$ -Function is that the initial conditions are inserted in the expansion. The set of the two arbitrary constants the any second order differential equation is included in the own definition of the function. One is still used to see the initial conditions as independent of the function in the realm of linear differential equations. In the nonlinear case this is not always the case and the first example is provided by the nonlinear but integrable of the Elliptic Functions. Beyond these functions we have to deal with nonlinear but integrable second order differential equations as the Painlevé Transcendents that also enjoy the same property of expansions containing the initial conditions. They are functions whose the movable singularities are only poles.

2) An apparent generalization of the invariant giving rise to the above $W$-equation (remember that $W^{\prime}=\psi$ ) can be written as: 


$$
\frac{1}{2} \psi^{\prime 2}+\psi^{3}=a_{0} \psi^{2}+c_{1} \psi+c_{2}
$$

After deriving twice the invariant this becomes:

$$
\psi^{\prime \prime \prime}+6 \psi \psi^{\prime}=2 a_{0} \psi
$$

Indeed the case $a_{0}=0$ yields the case fully discussed in (1). For $a_{0}=2$ and $a_{0}=3$ we recover (26) and (31) of [3], which have also been solved using various algebraic and quite cumbersome and tedious procedures in this mentioned reference. However they are actually Weierstrass $\mathcal{P}(\xi)$-Functions if one remembers the well known property of the third order algebraic equations for which we can always eliminate the second degree term using a trivial translation of the dependent variable $\psi$ (see [14]).

\section{Conclusion}

In this note, we have shown the power of the method of the symmetries for extracting all the information in a Non Linear Partial Differential equations; not only for finding the analytical solutions as it is usually used but also the algebraic structure: The Lie Algebra and the subgroups of the Invariants are explicitly shown as well as its relations to well known kinematical Lie Algebras. Also the connection with Elliptic Functions given in the last paragraph is interesting in its own. Other solutions can also be found with the help of Painlevé Transcendents, but this research which is now in progress will be postponed for a future publication.

\section{Acknowledgements}

This research has been carried out with the partial support of MINECO under the project MAT2013-46308-C2-1-R and JCyL Project Number SA226U13.

\section{References}

[1] Bogoyavlenskii, O. (1990) Russian Mathematical Surveys, 45, 1-86.

[2] Peng, Y. (2006) International Journal of Theoretical Physics, 45, 1779-1783. https://doi.org/10.1007/s10773-006-9139-7

[3] Saleh, R., Kassem, M. and Mabrouk, S. (2017) Mathematical Methods in the Applied Sciences, 40, 5851-5862. https://doi.org/10.1002/mma.4435

[4] Wazwaz, A. (2008) Applied Mathematics and Computation, 196, 363-370. (See also 203, 592-597)

[5] Kumar, R. (2016) IOSR Journal of Mathematics, 12, 144-147. https://doi.org/10.9790/5728-120402144147

[6] Olver, P.J. (1986) Applications of Lie Groups to Differential Equations. 2nd Edition, Springer Verlag, Berlin.

[7] Weiss, J., Tabor, M. and Carnevale, G. (1983) Journal of Mathematical Physics, 24, 522-536. https://doi.org/10.1063/1.525721

[8] Weiss, J. (1983) Journal of Mathematical Physics, 24, 1405-1423. https://doi.org/10.1063/1.525875

[9] Estévez, P.G., Prada, J. and Villarroel, J. (2007) Journal of Physics A: Mathematical 
and Theoretical, 40, 7213-7231. https://doi.org/10.1088/1751-8113/40/26/008

[10] Hammermesh, M. (1964) Group Theory and its Applications to Physics Problems. 2nd Edition, Addison-Wesley Publishing Co., Inc., Boston.

[11] Cerveró, J.M. and Polo, P.P. (2016) European Journal of Physics, 37, 055401.

[12] Dilatations and the Conformal Group Are Treated in the Recent Report by $\mathrm{H}$. Dreyer. http://edu.itp.phys.ethz.ch/fs13/cft/CGIVD2_Dreyer.pdf

[13] Byrd, P.F. and Friedman, M.D. (1971) Handbook of Elliptic Functions for Engineers and Scientists. 2nd Edition, Springer Verlag, Berlin. https://doi.org/10.1007/978-3-642-65138-0

[14] Clarkson, P.A. (2006) The Painlevè Equations-Nonlinear Special Functions. In: Marcellán, F. and van Assche, W., Eds., Orthogonal Polynomials and Special Functions. Computation and Applications? Lecture Notes in Mathematics, 1883. Springer Verlag, Berlin, 331-411. 